\title{
Structure and Function of the Influenza A Virus Non-Structural Protein 1
}

\author{
Chang Woo $\mathrm{Han}^{1 \dagger}$, Mi Suk Jeong ${ }^{2 \dagger}$, and Se Bok Jang ${ }^{1 *}$ \\ ${ }^{1}$ Department of Molecular Biology, College of Natural Sciences, Pusan National University, Busan 46241, Republic of Korea \\ ${ }^{2}$ Korea Nanobiotechnology Center, Pusan National University, Busan 46241, Republic of Korea
}

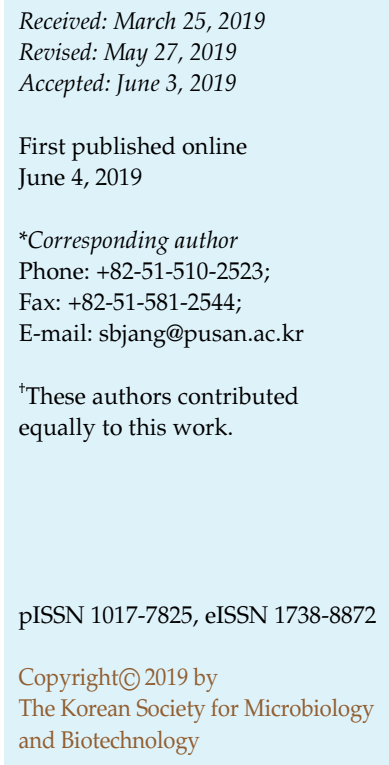

The influenza A virus is a highly infectious respiratory pathogen that sickens many people with respiratory disease annually. To prevent outbreaks of this viral infection, an understanding of the characteristics of virus-host interaction and development of an anti-viral agent is urgently needed. The influenza A virus can infect mammalian species including humans, pigs, horses and seals. Furthermore, this virus can switch hosts and form a novel lineage. This so-called zoonotic infection provides an opportunity for virus adaptation to the new host and leads to pandemics. Most influenza A viruses express proteins that antagonize the antiviral defense of the host cell. The non-structural protein 1 (NS1) of the influenza A virus is the most important viral regulatory factor controlling cellular processes to modulate host cell gene expression and double-stranded RNA (dsRNA)-mediated antiviral response. This review focuses on the influenza A virus NS1 protein and outlines current issues including the life cycle of the influenza A virus, structural characterization of the influenza A virus NS1, interaction between NS1 and host immune response factor, and design of inhibitors resistant to the influenza A virus.

Keywords: Influenza A virus, NS1, virus-host interaction, antiviral defense, inhibitor

\section{Fundamental Biology of Influenza Viruses}

Influenza viruses cause a seasonal epidemic that can occur on a regular basis, posing a major threat to world health [1]. For the influenza virus to infect an organism, the host interferon (IFN) response must be overcomed first [2]. Interferons and other inborn immune mediators have devastating impacts on the result of influenza virus infections in terms of disease severity, since highly pathogenic avian influenza (HPAI) viruses are often associated with excessive cytokine responses [3]. Synthesis, structure and intracellular localization are essential to investigations of influenza virus infection, host response, antiviral drugs and vaccines [4]. Early influenza virus infections should eliminate the virus by inducing efficient activation of the IFN system. Recent studies have focused on understanding the fundamental biology of influenza viruses to meet these needs.

Influenza viruses belong to the Orthomyxoviridae family and are classified as either type A, B, C, or the recently identified type D $[5,6]$. Influenza A viruses (IAVs) contain 8, negative-sense, single-stranded viral RNA (vRNA) gene segments, which encode transcripts for ten basic viral proteins, in addition, various strain-dependent accessory proteins [7]. The 8 genome segments of the influenza A virus have been shown to contain the genetic information for the following eight virus polypeptides: matrix1 (M1), matrix2 (M2), hemagglutinin (HA), neuraminidase (NA), nucleoprotein (NP), non-structural protein 1 (NS1), nonstructural protein 2 (NS2: also described as nuclear export protein, NEP), polymerase acidic protein (PA), polymerase basic protein 1 (PB1), polymerase basic protein 2 (PB2) and polymerase basic protein 1-F2 (PB1-F2) [8]. Viral protein, notably the non-structural protein (NS1), is generally regarded as the common factor by which most influenza A viruses antagonize host innate immune responses [9]. NS1 is encoded by viral segment 8 , which encodes the viral nuclear export protein NEP. In addition, the influenza A 
virus NS1 protein interacts with a variety of proteins to inhibit host cell immune responses and promote viral replication [10]. NS1 is important for multiple viral functions, including temporal adjustment of viral RNA synthesis, control of viral mRNA splicing, adjustment of virus particle morphogenesis, and suppression of host apoptotic response through the activation of phosphoinositide 3kinase (PI3K) [11-13]. It has also been shown to interact with replication intermediates of viral RNA to block these molecules from recognition by cellular pattern recognition receptors (PRRs) [14]. Although the RNA-binding and effector domains of NS1 structures have been identified, the studies related to their specific protein-protein interactions of the full-length Influenza virus A NS1 are still uncertain [15].

Non-conservative mutations have been shown to enhance or decrease the virulence of the H1N1 virus by slightly stabilizing or destabilizing the structural domain of the NS1 or NS1-dsRNA, thereby increasing or decreasing the function of the NS1 protein. Other strains of the NS2 protein caused non-conservative mutations in other regions and were slightly impaired in function [16]. In the infected cell, NS1 is localized in the nucleus and NS2 in the cytoplasm; hence, the virus function was slightly inhibited and consequently decreased the pathogenicity of the virus. Thus, these studies justify the structure-function relationships of the viral NS1 and NS2 proteins. Recently, many research groups have been identifying full-length NS1 structures or specific interaction proteins that can inhibit influenza A virus NS1. Therefore, understanding the full-length NS1 protein can facilitate investigation of the interaction between influenza A virus and antiviral response.

\section{Life Cycle of the Influenza A Virus}

When the influenza A virus enters the body, the influenza HA molecule recognizes sialic ( $\mathrm{N}$-acetylneuraminic) acid on the host cell surface. HA is a type I transmembrane glycoprotein exposed on the virus surface as a homotrimer, in which each monomer consists of two subunits, HA1 and HA2 $[17,18]$. In the low $\mathrm{pH}$ environment of the endosome, the cleaved HA with a fusogenic stalk domain of HA2 mediates fusion between the viral membrane and the endosomal membrane and permits entrance of viral ribonucleoprotein (vRNP) complex into the host cell. The vRNP complex consists of 8 negative-sense, single-stranded vRNA, nucleoprotein (NP) and the influenza A virus RNA polymerase (consisting of PA, PB1, and PB2) [19, 20] (Fig. 1). Subsequent fusion allows the vRNP complex to be released into the cellular cytoplasm, after which it enters the host nucleus by active transport. The nucleus is the where all influenza virus RNA synthesis takes place. To start the process of transcription, the virus RNA polymerase binds to the highly conserved and near complementary 13

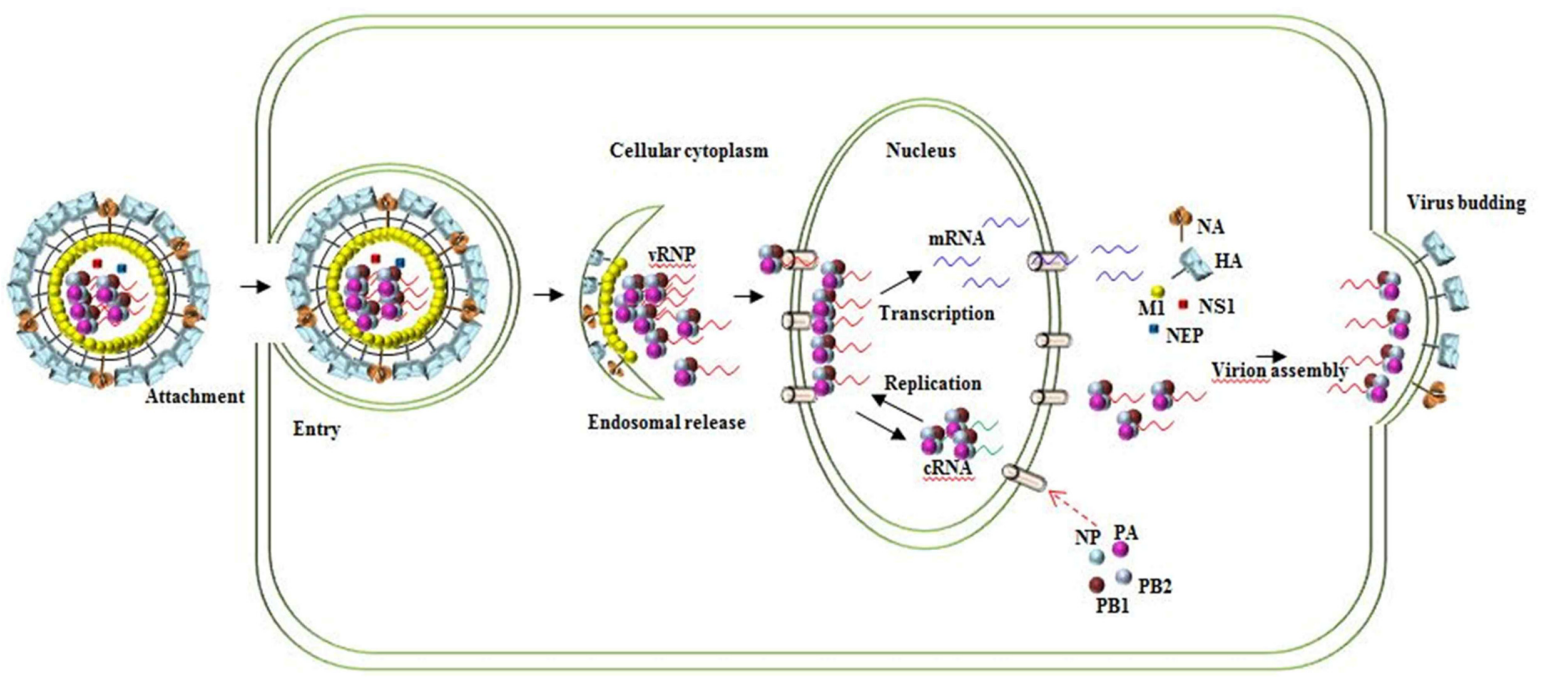

Fig. 1. Life cycle of the influenza A virus.

The hemagglutinin (HA) spike on the viral envelope binds to a sialic acid receptor on the surface membrane of the infected cell and is then endocytosed. The virus is exposed to the cytoplasm and the viral ribonucleoprotein (vRNP) complex is released and then transported into the host cell nucleus. The vRNP acts as an independent functional unit that directs the synthesis of two positive-sense RNAs (mRNA and cRNA) by using its negative-sense vRNA as a template. After transcription and processing, positive-sense mRNAs are exported and translated in the cytoplasm. Newly-formed influenza virus RNPs are transported to the cytoplasmic membrane, where they are packaged into budding virions. 
nucleotides at the $5^{\prime}$ end and 12 nucleotides at the $3^{\prime}$ end of all eight segments. However, the influenza virus polymerase does not have inherent capping activity and it synthesizes viral messenger RNAs using the 5' cap of host pre-mRNAs derived from cellular transcripts by a unique 'capsnatching' mechanism that is mediated by the PB1 and PB2 proteins [21, 22]. Its replication mechanism is primerindependent and proceeds via a complementary RNA (cRNA) replicative intermediate [23]. Mature viral mRNAs are exported to the cytoplasm by the viral proteins M1 and NEP/NS2 and translated like host mRNA [24, 25]. Next, viral HA, NA, and M2 proteins are synthesized on the rough endoplasmic reticulum and matured in the Golgi complex. Finally, the formation of mature virus particles (virions) and budding occur at the plasma membrane [26]. In the later stage of the viral replication cycle, nonstructural NS1 protein affects the morphogenesis of viral particles, but it is not a structural component of the viral particles [27].

\section{Structural Characterization of the NS1 Protein}

The non-structural protein 1 (NS1) is a crucial influenza A virus immune regulator that antagonizes antiviral response. The NS1 protein counteracts the production of IFNs and the activities of IFN-induced proteins that restrict influenza virus replication [28]. Furthermore, NS1 is a multifunctional regulatory viral protein that plays a critical role as a posttranscriptional regulatory factor in the life cycle of influenza virus; specifically, binding virion RNA [29, 30]. NS1 protein comprises 215-237 amino acids (aa) relying on the strain, and has a molecular weight of about $26 \mathrm{kDa}$ [31]. NS1 protein consists of 2 distinct functional domains connected by a flexible linker region (LR): an N-terminal RNAbinding domain (RBD, aa 1-73) and a C-terminal effector domain (ED, aa 74-207) (Fig. 2A). The nucleotide sequence (NLS) (aa 35-41) overlaps the sequences needed for dsRNA binding [32]. The NS1 protein can conduct functions in both the nucleus and cytoplasm. The sequences of NS1 proteins from independent virus strains are compared. H3N2 has sequence identities of $66.96 \%$ with $\mathrm{H} 5 \mathrm{~N} 1$ and $91.30 \%$ with $\mathrm{H} 2 \mathrm{~N} 2$. In addition, $\mathrm{H} 3 \mathrm{~N} 2$ has sequence identities of $84.78 \%$ with H1N1 and $76.04 \%$ with H7N9. The highest sequence variations appear in the C-terminal region of the ED (Fig. 2B).

The NS1 full-length is found as a homodimer in vitro with both the RNA-binding and effector domains bringing to dimerization [33]. The dimeric NS1 binds to the dsRNA duplex with a 1:1 stoichiometry [34]. The RBD is a six- helical symmetrical arrangement with each monomer consisting of three $\alpha$-helices (Fig. 2C). Each monomer aligns to organize antiparallel strands configured with many conserved hydrophilic and basic amino acids, including the critical amino acids Arg 38 and Lys 41, which interact directly with the dsRNA [35]. In addition, bioinformatics and protein structural studies about the interface between the two monomers suggest that the Nterminal RBD forms a highly stable homodimer. The monomer of the c-terminal ED is comprised of $3 \alpha$-helices and $7 \beta$-strands. The $\beta$-strands form an antiparallel twisted $\beta$-sheet surrounding one part of the central $\alpha 3$ long helix, which is held in place through an extensive network of hydrophobic interactions between the twisted $\beta$-sheet and the $\alpha$-helix. The ED of NS1 from the influenza A virus also forms weak dimers in solution, with a helix-helix interaction mediated by tryptophan 187 [36]. This ED dimerization contributes to cooperativity in binding dsRNA by fulllength NS1 proteins, which probably only occurs efficiently via cooperative binding of NS1 multimers.

In addition, the binding sites for the $30-\mathrm{kDa}$ subunit of cleavage and polyadenylation specificity factor (CPSF 30) are near the ED of NS1 protein, and the CPSF 30 binding sites are located in the 144 and 186 amino acid regions. The NS1 protein possesses the sequence ${ }^{226} \mathrm{ARSK}^{229}$ at its ED that is similar to the sequence ${ }^{1} \mathrm{ARTK}^{4}$ at the N-terminal of histone $\mathrm{H} 3$. Analogous to the histone H3K4 sequence, host enzymes methylate and acetylate the terminal lysine in the ${ }^{226} \mathrm{ARSK}^{229}$ sequence of the NS1 in vivo and in vitro. The methylated NS1 mimics H3 and binds to a specific transcription factor, polymerase-associated factor 1 complex (PAF1C) and chromodomain helicase DNA-binding protein 1 (CHD1), which results in inhibition of inducible expression of specific genes containing host antiviral genes. Influenza A virus strains circulating in humans differ in the capability of the NS1 protein to inhibit the activation of interferon-beta transcription and IRF3. The C-terminal difference of NS1 ED accounts for various functional differences among the NS1 proteins having different viral strains. The C-terminal region of ED is widely involved in giving to the IFN-b and IRF3 transcriptional phenotypes of the NS1 protein [37]. The homology of the amino acid at this site of the 196 position is closely related to the IFN-b and IRF3 transcriptional phenotypes of the NS1. The NS1 protein, which does not inhibit the activation of IFN-b and IRF3 transcription, contains $\mathrm{K}$ at this position, while the NS1 protein that inhibits this activation contains $\mathrm{E}$ at this position. 
A

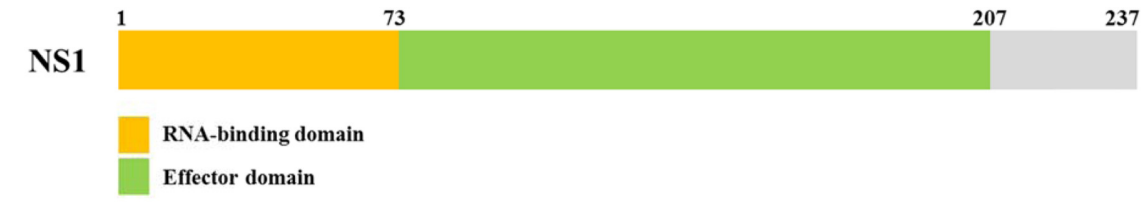

B

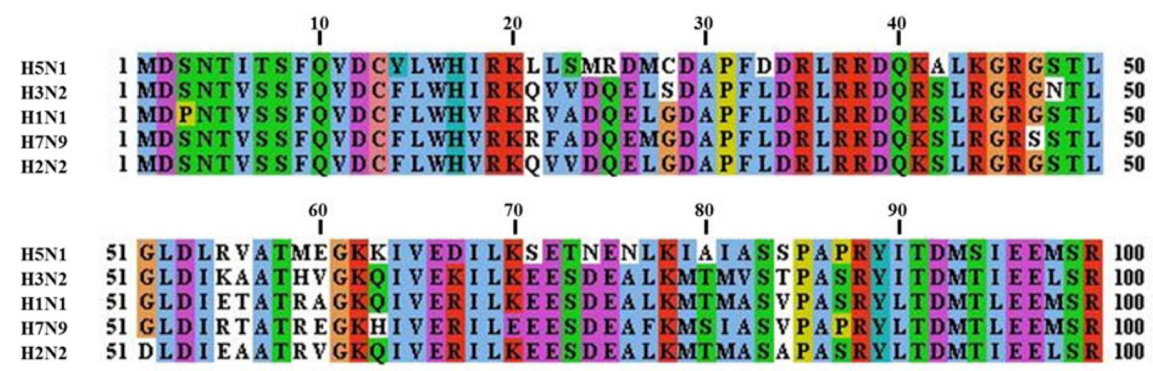

H2N2 51 DLDI IEATRVGKO I VERILLEESDEALKMTMASAPASRYLTDMT IEELSR 100

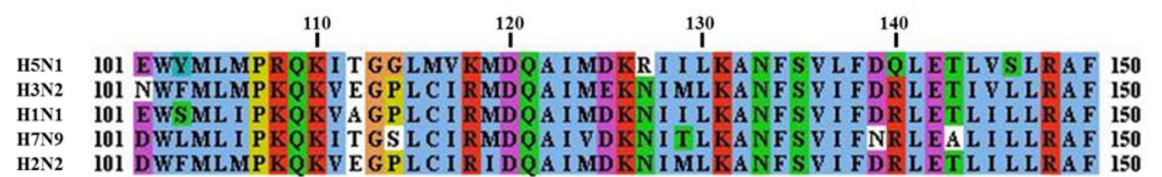

H2N2 101 DWFMLMPKQKVEGP LC I R I DQQA IMD KNIMLK A NFS V I F DR LETLILLRA F 150

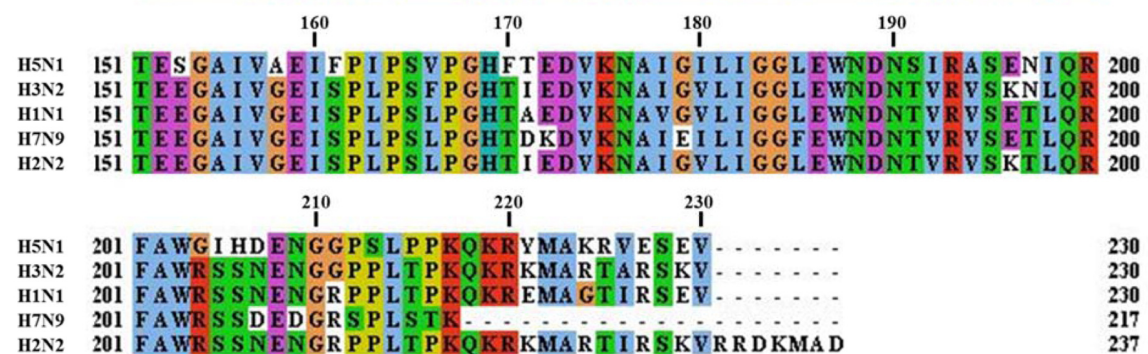

C

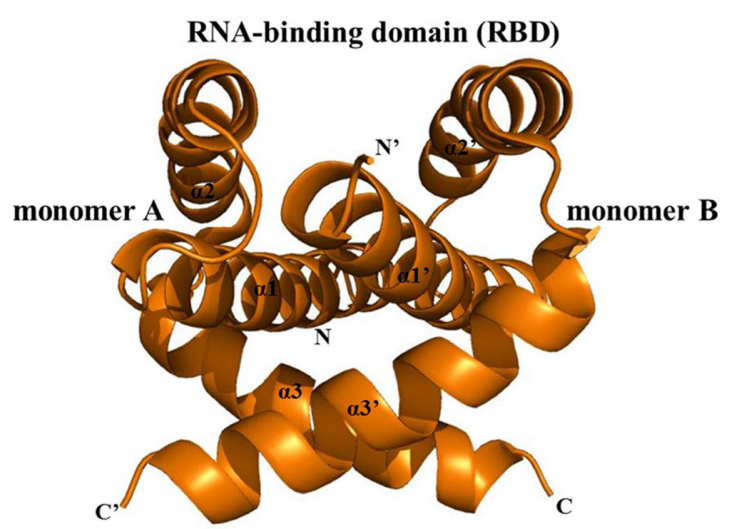

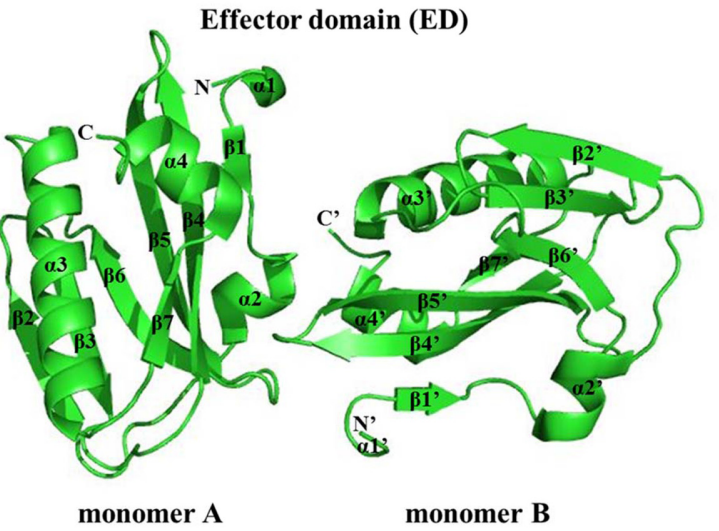

Fig. 2. Structure of the non-structural protein 1 (NS1) domain.

NS1 is composed of N-terminal RNA-binding domain (RBD, amino acids 1-73) and C-terminal effector domain (ED, amino acids 74-207). (B) The sequence of NS1 proteins from different virus strains is largely conserved. (C) Schematic ribbon diagrams of the RNA-binding domain (PDB ID, 2N74) and effector domain (PDB ID, 3EE8).

\section{Interaction between NS1 and Host Immune Response Factors}

The influenza A virus NS1 protein is expressed at very high levels in infected host cells. The mRNA that is transcribed from viral RNA segment 8 either encodes NS1, if unspliced, or nuclear export protein after being spliced [38]. The NS1 protein is known to bind to double-stranded 


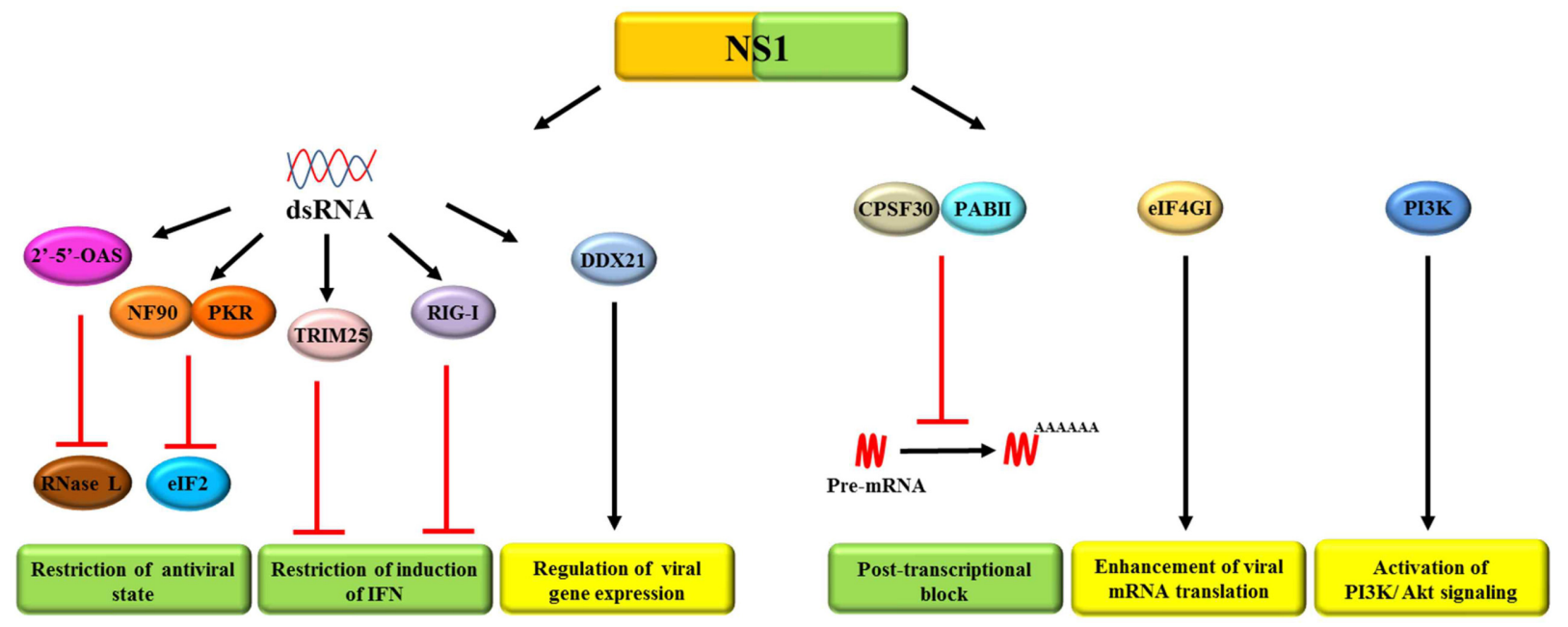

Fig. 3. The network of the host factors that interacts with the NS1 protein of influenza A virus.

The N-terminal RNA-binding domain (RBD) of NS1 binds to the host cellular proteins through double-stranded RNA (dsRNA). RBD binds to the antiviral enzymes, including 2'-5'-oligoadenylate synthetase (2'-5'-OAS)/RNase L and nuclear factor 90 (NF90)-protein kinase R (PKR)/ eukaryotic translation inhibitor factor 2 and inhibit the antiviral state. RBD interacts with E3 ubiquitin ligase tripartite motif-containing 25 (TRIM25) and retinoic-acid-inducible protein 1 (RIG-I), limiting the induction of RIG-I-mediated type I IFN (interferon). In addition, the interaction of RBD of the NS1 with RNA helicase DDX21 leads to regulation of viral gene expression. The C-terminal effector domain (ED) of NS1 interacts with the 30-kDa subunit of cellular cleavage (CPSF30)/poly (A)-binding protein II (PABII), eukaryotic translation initiation factor 4GI (eIF4GI), and phosphatidylinositol 3-kinase (PI3K)/protein kinase B (Akt). Each of these interactions provide post-transcriptional blocks to cellular mRNA maturation, enhancement of viral mRNA translation, and PIK3/Akt signaling activation.

RNA via N-terminal RNA-binding domain and it specifically binds to the region of the RNA containing either 5'- or 3'terminal common genomic sequence [39]. The binding of influenza A virus NS1 protein to dsRNA protects the virus against the antiviral state induced by interferon- $\alpha / \beta$ (IFN$\alpha / \beta)$, primarily by blocking activation of the $2^{\prime}-5^{\prime}-$ oligoadenylate synthetase (OAS) / RNase L pathway (Fig. 3) $[40,41]$. Furthermore, the NS1 protein interacts with protein kinase R (PKR) to counteract PKR-induced eukaryotic translation inhibitor factor 2 (eIF2) [42]. Recently, NF90 was found to be involved in host antiviral mechanisms targeting various viruses, [43] regulates PKR activation and stress granule formation in influenza A virus-infected cells and is an interacting protein of influenza A virus NS1 [44]. NF90 displays its antiviral activity by matching the inhibitory role of NS1 on PKR phosphorylation. In addition, the NS1 RBD binds to E3 ubiquitin ligase tripartite motifcontaining 25 (TRIM25) and inhibits ubiquitination of retinoic-acid-inducible protein 1 (RIG-I), which reduces the induction of RIG-I-mediated type I IFN [45]. RIG-I and melanoma differentiation-associated proteins (MDA-5) each recognize variant types of viral RNA. RIG-I is most effectively activated by shorter stretches of $5^{\prime}$-triphosphate or 5'-diphosphate dsRNA while MDA-5 is activated by long stretches of dsRNA in a $5^{\prime}$ phosphate-independent manner. Thus, the pathogen-recognition receptors have been seen to recognize variant RNA virus families [46, 47]. Knockdown research studies have demonstrated that IFN induction against influenza virus infection is majorly regulated by RIG-I. Influenza virus-induced IFN expression in the key site of influenza virus replication and airway epithelial cells is RIG-I-dependent [48]. However, MDA-5 may donate to IFN induction by influenza viruses, because small reductions in IFN induction and IFN-stimulated genes responses can be shown in infected MDA-5-deficient cells compared to control cells. In addition, IFNs are still expressed in the next infection with influenza virus or transfections with influenza RNA in chicken cells, which lack RIG-I. Alternatively, IFN induction in this process is adjusted by MDA-5 [49-51]. RNA synthesis of the influenza A virus is catalyzed by a viral polymerase consisting of PA, PB1 and PB2. Recent results have shown that host DDX21 RNA helicase binds to PB1 and inhibits polymerase assembly to limit influenza A virus, thereby reducing viral RNA and protein synthesis [52]. DDX21 binds to the NS1 $\mathrm{N}$-terminal region, which as well joins in other important functions. Viral mutants in which the NS1 protein cannot bind to DDX21 reduce viral protein synthesis in the early 
and late steps of infection, and when DDX21 is knocked out, the phenotype is converted to the wild type.

Another domain, the C-terminal effector domain of the influenza A virus NS1 protein, interacts with several host factors. Specifically, the NS1 protein provides the critical function of blocking the processing of 3 '-terminal cellular or viral pre-mRNAs by binding two cellular proteins: CPSF30 and poly (A)-binding protein II (PABII). As a result of the sequestering of CPSF30 by the NS1 protein, unprocessed cellular pre-mRNAs accumulate in the nucleus, and cellular mRNA production in the cytoplasm is inhibited [53, 54]. In addition, NS1 preferentially enhances translation of the influenza virus mRNA by recruiting eukaryotic translation initiation factor 4GI (eIF4GI), the large subunit of the cap-binding complex eIF4F, specifically to its $5^{\prime}$-untranslated region ( $5^{\prime} \mathrm{UTR}$ ) [55]. Furthermore, the NS1 has been required in control to the phosphatidylinositol 3-kinase (PI3K) activation by binding the PI3K p $85 \beta$ subunit, resulting in the activation of $\mathrm{PI} 3 \mathrm{~K} /$ protein kinase $\mathrm{B}(\mathrm{Akt})$ signaling [36]. Thus, influenza A virus NS1 protein inhibits or activates signaling pathways to insure effective virus replication. Other recent studies have concentrated on the interaction of NS1 mutants with host immune response factors. Mutations of the NS1 protein have been shown to impact on host cell IFN responses and the reduced capability of the NS1 mutant viruses to induce IFN gene. The protein expression is associated with reduced activation of STAT1 and a lower level of ISG expression [10]. The results were demonstrated by mutation at the position 171 in the NS1 resulting in reduced expression of IFN and ISGs by A549 cells.

\section{Inhibitor Development against Influenza A Virus NS1}

One of the inhibitors reported, JJ3297, is effected on NS1induced inhibition of IFN mRNA production, and it depends strictly on the presence of RNase $\mathrm{L}$. The action of JJ3297 is insensitive to other IFN signaling pathway elements such as RIG-I [56]. In another study, several chemical compounds inhibited both influenza virus replication and NS1 function. The compounds (NSC95676, NSC109834, and NSC128164) launched a considerable reduction in viral NS1-specific RNAs (NS1 and M2). However, the infected cells by influenza A NS1 gene knockout viruses (delNS1) did not show the same decrease in overall viral RNA levels. The compounds are active only in the existence of NS1 [57]. Another study emphasized that interference with the interaction between NS1 and CPSF30 is a research target. The second and third zinc fingers (F2F3) domain of CPSF30 binds efficiently to the NS1 protein. Interestingly, cellular expression of a small fragment of CPSF30 sufficient to bind NS1 has been shown to inhibit virus replication and increase production of IFN- $\beta$ mRNA [58].

Petit et al. have described the binding site and inhibition mechanism of two small-molecule experimental inhibitors of influenza A viruses in detail [59]. They showed that the inhibitors, A9 and A22, interacted with just one of the two separately folded domains of NS1, the NS1 effector domain. In addition, the structures of the antiviral compounds were highly analogous to a fraction of a host CPSF30 protein that the NS1 ED binds to short-circuit the body's immune response. Thus, A9 and A22 exhibited to inhibit influenza viral replication and NS1 function by interrupting with the interaction between CPSF30 and the NS1 effector domain. Other biological studies have shown that Arg 38 of the RBD interacts with dsRNA directly. For example, the influenza A/Udorn/307/72 virus (H3N2) that encodes an R38A NS1 protein is very attenuated, suggesting that small molecule drugs that interrupt NS1-dsRNA binding should block viral replication [60]. Accordingly, the NS1-dsRNA interaction is a prospective target for inhibition of the small molecule drugs.

Much progress has been achieved in studies of the influenza virus. However, the influenza virus continues to be a major health threat, especially with respect to the NS1 protein, which is a potent toxic factor that antagonizes host immune response. Recently, several studies have also seen that NS1 affects the morphogenesis of the virus particle. For this reason, research groups are searching for small molecules or peptides that can inhibit NS1 [56, 57]. Although some inhibitors have been developed, there are still many problems that need to be addressed. Every year, influenza viruses kill 250,000 to 500,000 people all over the world. The virus is known to quickly evolve to bring about pandemic strains that few people have immunity to resist. Viral resistance has hindered the effectiveness of a few initial antiviral agents that were worked up to cure influenza; therefore, there is a great need for novel antiviral compounds. In this review, the crucial role of the influenza A virus NS1 protein in negative regulation of innate antiviral responses is explained and the need for better insight into the function of NS1 protein is suggested. A more complete understanding of the structure and function of this influenza A virus NS1 protein will be applicable to the development of new anti-influenza therapies. 


\section{Acknowledgments}

This study was supported by the Basic Science Research Program through the National Research Foundation of Korea (NRF) funded by the Ministry of Education, Science and Technology (2018R1D1A1B07043701) to S.B.J. and (2016R1D1A1B02011142) to M.S.J.

\section{Conflict of Interest}

The authors have no financial conflicts of interest to declare.

\section{References}

1. Gostin LO. 2004. Pandemic Influenza: public health preparedness for the next global health emergency. J. Law Med. Ethics 32: 565-573.

2. Killip MJ, Fodor E, Randall RE. 2015. Influenza virus activation of the interferon system. Virus Res. 209: 11-22.

3. Baskin CR, Bielefeldt-Ohmann H, Tumpey TM, Sabourin PJ, Long JP, Garcia-Sastre A, et al. 2009. Early and sustained innate immune response defines pathology and death in nonhuman primates infected by highly pathogenic influenza virus. Proc. Natl. Acad. Sci. USA 106: 3455-3460.

4. Watanabe T, Kawakami E, Shoemaker JE, Lopes TJ, Matsuoka Y, Tomita Y, et al. 2014. Influenza virus-host interactome screen as a platform for antiviral drug development. Cell Host Microbe 16: 795-805.

5. Hause BM, Collin EA, Liu R, Huang B, Sheng Z, Lu W, et al. 2014. Characterization of a novel influenza virus in cattle and Swine: proposal for a new genus in the Orthomyxoviridae family. MBio 5: e00031-00014.

6. Palese P, Schulman JL. 1976. Mapping of the influenza virus genome: identification of the hemagglutinin and the neuraminidase genes. Proc. Natl. Acad. Sci. USA 73: 21422146.

7. McGeoch D, Fellner P, Newton C. 1976. Influenza virus genome consists of eight distinct RNA species. Proc. Natl. Acad. Sci. USA 73: 3045-3049.

8. Dou D, Revol R, Ostbye H, Wang H, Daniels R. 2018. Influenza A Virus Cell Entry, Replication, Virion Assembly and Movement. Front. Immunol. 9: 1581.

9. Marc D. 2014. Influenza virus non-structural protein NS1: interferon antagonism and beyond. J. Gen. Virol. 95: 25942611.

10. Plant EP, Ilyushina NA, Sheikh F, Donnelly RP, Ye Z. 2017. Influenza virus NS1 protein mutations at position 171 impact innate interferon responses by respiratory epithelial cells. Virus Res. 240: 81-86.

11. Alonso-Caplen FV, Krug RM. 1991. Regulation of the extent of splicing of influenza virus NS1 mRNA: role of the rates of splicing and of the nucleocytoplasmic transport of NS1 mRNA. Mol. Cell Biol. 11: 1092-1098.

12. Skorko R, Summers DF, Galarza JM. 1991. Influenza A virus in vitro transcription: roles of NS1 and NP proteins in regulating RNA synthesis. Virology 180: 668-677.

13. Ehrhardt C, Wolff T, Ludwig S. 2007. Activation of phosphatidylinositol 3-kinase signaling by the nonstructural NS1 protein is not conserved among type A and B influenza viruses. J. Virol. 81: 12097-12100.

14. Wilkins C, Gale M, Jr. 2010. Recognition of viruses by cytoplasmic sensors. Curr. Opin. Immunol. 22: 41-47.

15. Carrillo B, Choi JM, Bornholdt ZA, Sankaran B, Rice AP, Prasad BV. 2014. The influenza A virus protein NS1 displays structural polymorphism. J. Virol. 88: 4113-4122.

16. Salahuddin P, Khan AU. 2010. Structural and functional analysis of NS1 and NS2 proteins of H1N1 subtype. Genomics Proteomics Bioinformatics 8: 190-199.

17. Righetto I, Milani A, Cattoli G, Filippini F. 2014. Comparative structural analysis of haemagglutinin proteins from type A influenza viruses: conserved and variable features. BMC Bioinformatics 15: 363.

18. Mair CM, Ludwig K, Herrmann A, Sieben C. 2014. Receptor binding and $\mathrm{pH}$ stability - how influenza A virus hemagglutinin affects host-specific virus infection. Biochim. Biophys. Acta. 1838: 1153-1168.

19. Zheng W, Tao YJ. 2013. Structure and assembly of the influenza A virus ribonucleoprotein complex. FEBS Lett. 587: 1206-1214.

20. Hamilton BS, Whittaker GR, Daniel S. 2012. Influenza virusmediated membrane fusion: determinants of hemagglutinin fusogenic activity and experimental approaches for assessing virus fusion. Viruses 4: 1144-1168.

21. Dias A, Bouvier D, Crepin T, McCarthy AA, Hart DJ, Baudin $\mathrm{F}$, et al. 2009. The cap-snatching endonuclease of influenza virus polymerase resides in the PA subunit. Nature 458: 914-918.

22. Te Velthuis AJ, Fodor E. 2016. Influenza virus RNA polymerase: insights into the mechanisms of viral RNA synthesis. Nat. Rev. Microbiol. 14: 479-493.

23. York A, Hengrung N, Vreede FT, Huiskonen JT, Fodor E. 2013. Isolation and characterization of the positive-sense replicative intermediate of a negative-strand RNA virus. Proc. Natl. Acad. Sci. USA 110: E4238-4245.

24. Bui M, Wills EG, Helenius A, Whittaker GR. 2000. Role of the influenza virus M1 protein in nuclear export of viral ribonucleoproteins. J. Virol. 74: 1781-1786.

25. O'Neill RE, Talon J, Palese P. 1998. The influenza virus NEP (NS2 protein) mediates the nuclear export of viral ribonucleoproteins. EMBO J. 17: 288-296.

26. Nayak DP, Balogun RA, Yamada H, Zhou ZH, Barman S. 2009. Influenza virus morphogenesis and budding. Virus Res. 143: 147-161. 
27. Garaigorta U, Falcon AM, Ortin J. 2005. Genetic analysis of influenza virus NS1 gene: a temperature-sensitive mutant shows defective formation of virus particles. J. Virol. 79: 15246-15257.

28. Price GE, Gaszewska-Mastarlarz A, Moskophidis D. 2000. The role of alpha/beta and gamma interferons in development of immunity to influenza A virus in mice. J. Virol. 74: 39964003.

29. Hatada E, Saito S, Okishio N, Fukuda R. 1997. Binding of the influenza virus NS1 protein to model genome RNAs. J. Gen. Virol. 78 (Pt 5): 1059-1063.

30. Marion RM, Aragon T, Beloso A, Nieto A, Ortin J. 1997. The N-terminal half of the influenza virus NS1 protein is sufficient for nuclear retention of mRNA and enhancement of viral mRNA translation. Nucleic Acids Res. 25: 4271-4277.

31. Hale BG, Randall RE, Ortin J, Jackson D. 2008. The multifunctional NS1 protein of influenza A viruses. J. Gen. Virol. 89: 2359-2376.

32. Melen K, Kinnunen L, Fagerlund R, Ikonen N, Twu KY, Krug RM, et al. 2007. Nuclear and nucleolar targeting of influenza A virus NS1 protein: striking differences between different virus subtypes. J. Virol. 81: 5995-6006.

33. Nemeroff ME, Qian XY, Krug RM. 1995. The influenza virus NS1 protein forms multimers in vitro and in vivo. Virology 212: $422-428$.

34. Chien CY, Xu Y, Xiao R, Aramini JM, Sahasrabudhe PV, Krug RM, et al. 2004. Biophysical characterization of the complex between double-stranded RNA and the N-terminal domain of the NS1 protein from influenza A virus: evidence for a novel RNA-binding mode. Biochemistry 43: 1950-1962.

35. Talon J, Horvath CM, Polley R, Basler CF, Muster T, Palese P, et al. 2000. Activation of interferon regulatory factor 3 is inhibited by the influenza A virus NS1 protein. J. Virol. 74: 7989-7996.

36. Hale BG, Jackson D, Chen YH, Lamb RA, Randall RE. 2006. Influenza A virus NS1 protein binds p85beta and activates phosphatidylinositol-3-kinase signaling. Proc. Natl. Acad. Sci. USA 103: 14194-14199.

37. Kuo RL, Zhao C, Malur M, Krug RM. 2010. Influenza A virus strains that circulate in humans differ in the ability of their NS1 proteins to block the activation of IRF3 and interferon-beta transcription. Virology 408: 146-158.

38. Huang X, Zheng M, Wang P, Mok BW, Liu S, Lau SY, et al. 2017. An NS-segment exonic splicing enhancer regulates influenza A virus replication in mammalian cells. Nat. Commun. 8: 14751.

39. Cheng A, Wong SM, Yuan YA. 2009. Structural basis for dsRNA recognition by NS1 protein of influenza A virus. Cell Res. 19: 187-195.

40. Drappier M, Michiels T. 2015. Inhibition of the OAS/RNase L pathway by viruses. Curr. Opin. Virol. 15: 19-26.

41. Tisoncik JR, Billharz R, Burmakina S, Belisle SE, Proll SC, Korth MJ, et al. 2011. The NS1 protein of influenza A virus suppresses interferon-regulated activation of antigenpresentation and immune-proteasome pathways. J. Gen. Virol. 92: 2093-2104.

42. Li S, Min JY, Krug RM, Sen GC. 2006. Binding of the influenza A virus NS1 protein to PKR mediates the inhibition of its activation by either PACT or double-stranded RNA. Virology 349: 13-21.

43. Patino C, Haenni AL, Urcuqui-Inchima S. 2015. NF90 isoforms, a new family of cellular proteins involved in viral replication? Biochimie 108: 20-24.

44. Li T, Li X, Zhu W, Wang H, Mei L, Wu S, et al. 2016. NF90 is a novel influenza A virus NS1-interacting protein that antagonizes the inhibitory role of NS1 on PKR phosphorylation. FEBS Lett. 590: 2797-2810.

45. Gack MU, Albrecht RA, Urano T, Inn KS, Huang IC, Carnero E, et al. 2009. Influenza A virus NS1 targets the ubiquitin ligase TRIM25 to evade recognition by the host viral RNA sensor RIG-I. Cell Host Microbe 5: 439-449.

46. Kato H, Takeuchi O, Sato S, Yoneyama M, Yamamoto M, Matsui K, et al. 2006. Differential roles of MDA5 and RIG-I helicases in the recognition of RNA viruses. Nature 441: 101105.

47. Loo YM, Fornek J, Crochet N, Bajwa G, Perwitasari O, Martinez-Sobrido L, et al. 2008. Distinct RIG-I and MDA5 signaling by RNA viruses in innate immunity. J. Virol. 82: 335-345.

48. Le Goffic R, Pothlichet J, Vitour D, Fujita T, Meurs E, Chignard M, et al. 2007. Cutting Edge: Influenza A virus activates TLR3-dependent inflammatory and RIG-I-dependent antiviral responses in human lung epithelial cells. J. Immunol. 178: 3368-3372.

49. Husser L, Alves MP, Ruggli N, Summerfield A. 2011. Identification of the role of RIG-I, MDA- 5 and TLR3 in sensing RNA viruses in porcine epithelial cells using lentivirus-driven RNA interference. Virus Res. 159: 9-16.

50. Karpala AJ, Stewart C, McKay J, Lowenthal JW, Bean AG. 2011. Characterization of chicken Mda5 activity: regulation of IFN-beta in the absence of RIG-I functionality. J. Immunol. 186: $5397-5405$.

51. Liniger M, Summerfield A, Zimmer G, McCullough KC, Ruggli N. 2012. Chicken cells sense influenza A virus infection through MDA5 and CARDIF signaling involving LGP2. J. Virol. 86: 705-717.

52. Chen G, Liu CH, Zhou L, Krug RM. 2014. Cellular DDX21 RNA helicase inhibits influenza A virus replication but is counteracted by the viral NS1 protein. Cell Host Microbe 15: 484-493.

53. Nemeroff ME, Barabino SM, Li Y, Keller W, Krug RM. 1998. Influenza virus NS1 protein interacts with the cellular $30 \mathrm{kDa}$ subunit of CPSF and inhibits 3'end formation of cellular pre-mRNAs. Mol. Cell. 1: 991-1000. 
54. Chen Z, Li Y, Krug RM. 1999. Influenza A virus NS1 protein targets poly(A)-binding protein II of the cellular 3'end processing machinery. EMBO J. 18: 2273-2283.

55. Aragon T, de la Luna S, Novoa I, Carrasco L, Ortin J, Nieto A. 2000. Eukaryotic translation initiation factor 4GI is a cellular target for NS1 protein, a translational activator of influenza virus. Mol. Cell Biol. 20: 6259-6268.

56. Walkiewicz MP, Basu D, Jablonski JJ, Geysen HM, Engel DA. 2011. Novel inhibitor of influenza non-structural protein 1 blocks multi-cycle replication in an RNase L-dependent manner. J. Gen. Virol. 92: 60-70.

57. Basu D, Walkiewicz MP, Frieman M, Baric RS, Auble DT, Engel DA. 2009. Novel influenza virus NS1 antagonists block replication and restore innate immune function. $J$. Virol. 83: 1881-1891.

58. Twu KY, Noah DL, Rao P, Kuo RL, Krug RM. 2006. The CPSF30 binding site on the NS1A protein of influenza A virus is a potential antiviral target. J. Virol. 80: 3957-3965.

59. Kleinpeter AB, Jureka AS, Falahat SM, Green TJ, Petit CM. 2018. Structural analyses reveal the mechanism of inhibition of influenza virus NS1 by two antiviral compounds. J. Biol. Chem. 293: 14659-14668.

60. Min JY, Krug RM. 2006. The primary function of RNA binding by the influenza A virus NS1 protein in infected cells: Inhibiting the $2^{\prime}-5^{\prime}$ oligo (A) synthetase/RNase L pathway. Proc. Natl. Acad. Sci. USA 103: 7100-7105. 